\title{
Association of the First Antipsychotic Treatment Duration With the Re-Initiation of Treatment in Schizophrenia: A National Health Insurance Data-Based Study
}

\author{
Minho Song, MD, Jungsun Lee, MD, PhD, Harin Kim, MD, Soojin Ahn, MD, \\ Young Jae Choi, MD, Young Tak Jo, MD, and Sung Woo Joo, MD \\ Department of Psychiatry, Asan Medical Center, University of Ulsan College of Medicine, Seoul, Korea
}

\begin{abstract}
Objectives: The optimal duration of maintenance treatment for patients with first-episode schizophrenia (FES) remains unclear. We examined the first antipsychotic treatment duration and its association with re-initiation of treatment using a nationwide claim database.

Methods: Data from the Health Insurance Review and Assessment Service database in South Korea for 2007-2016 were used. Linear regression analysis and Cox proportional hazard models were used to evaluate the associations between the duration of the first antipsychotic treatment, time to re-initiation of treatment, and occurrence of treatment re-initiation.

Results: Of 30,143 patients with FES, $80.4 \%(n=24,231)$ received $<2$ years of the first antipsychotic treatment. In patients who discontinued treatment $(n=23,030)$, the rate of treatment re-initiation was $74.2 \%(n=17,086)$. As the duration of the first antipsychotic treatment increased, the time to re-initiation of treatment decreased $(\beta=-0.146, p<0.001)$; however, the rate of treatment reinitiation was relatively constant (hazard ratio $=1.001, \mathrm{p}<0.001$ ).

Conclusion: Long-term antipsychotic treatment was not significantly associated with the rate of treatment re-initiation but showed a negative association with the time to re-initiation of treatment. Further research is needed to better understand the optimal treatment duration for FES. (Korean J Schizophr Res 2021;24:60-67)
\end{abstract}

Keywords: Antipsychotic treatment; Re-initiation of treatment; Schizophrenia.

\section{Introduction}

Schizophrenia is a psychiatric disorder with an estimated lifetime prevalence of approximately $1 \%$ and leads to cognitive devastation and social maladjustment. ${ }^{1,2)}$ Antipsychotic drug plays a crucial role in the treatment of schizophrenia. Previous studies have reported that after the stabilization of symptoms for $1-2$ years, $57 \%-98 \%$ of the patients with firstepisode schizophrenia experience relapse after discontinuation or reduction in dose of antipsychotics. ${ }^{3-7)}$ Relapse is associated with an increased risk of self-harm or violence and poses a detrimental effect on interpersonal relationships and occupational function. ${ }^{2,8)}$ Recurrent relapses, usually defined as aggravation of psychotic symptoms, may indicate progression of the disease and hinder patients from recovering to their

Received: August 22, 2021 / Revised: September 23, 2021

Accepted: September 29, 2021

Address for correspondence: Sung Woo Joo, Department of Psychiatry, Asan Medical Center, University of Ulsan College of Medicine, 88 Olympicro 43-gil, Songpa-gu, Seoul 05505, Korea

Tel: 02-3010-3410, Fax: 02-485-8381

E-mail: jootak01@gmail.com previous level of functioning. ${ }^{9,10)}$ Therefore, several studies have been conducted to prevent relapse and identify risk factors associated with relapse, including caregiver criticism, substance use disorder, low treatment compliance, and poor premorbid adjustment. ${ }^{11)}$

Many studies have reported that the continuation of antipsychotic treatment is essential to prevent relapse $\mathrm{e}^{12-14)}$ and improve long-term outcomes ${ }^{15)}$ in first-episode schizophrenia, along with inadequate effectiveness of intermittent treatment. ${ }^{12,16)}$ Although it has been well acknowledged that the discontinuation of antipsychotic treatment is highly predictive of relapse in first-episode schizophrenia, the optimal maintenance period in terms of a balance between the prevention of relapse and adverse effects owing to a long-term use of antipsychotics, such as obesity and metabolic syndrome ${ }^{17,18)}$ remains unclear. A recent systematic review including six randomized control trials (RCTs) showed no correlation of the duration of maintenance treatment with relapse rate in firstepisode schizophrenia. ${ }^{19)}$ However, the findings need to be replicative and verified because of the limitations which were different treatment durations and short observation periods 
of the included RCTs. According to a recent systematic review of the treatment guidelines for schizophrenia, eleven of the twenty treatment guidelines mention the duration of maintenance treatment in first-episode schizophrenia. The treatment guidelines have tended to shift the antipsychotics discontinuation from "not recommended" to "partially recommended." Eight of the eleven guidelines specify the duration of maintenance treatment; seven guidelines recommend 1-2 years, while only one guideline recommends $2-5$ years. ${ }^{20}$

Current evidence on the discontinuation of antipsychotic treatment and its associated clinical outcomes in first-episode schizophrenia is largely based on the findings from RCTs. Despite the advantages of high intrinsic validity derived from random sampling and minimizing the risk of confounding bias, an RCT study design is limited by the selective study participants and a relatively short observation period. The patients who meet the strict inclusion criteria are more likely to have good compliance than those in actual clinical practice; therefore, the findings should be generalized with caution. ${ }^{21)}$ The disadvantages of RCT studies could serve as obstacles in performing the investigation on topics that require a large number of patients over a long period of observation.

A national health insurance system in the Republic of Korea covers the entire Korean population. The Health Insurance Review and Assessment (HIRA) Service reviews the claims generated by medical institutions for medical services used by the Korean population. The HIRA Service manages the claim database and has released them only for research purposes since 2015. The claim data include information on diagnostic codes, drug prescriptions, and hospitalization. The diagnostic code is based on the International Classification of Diseases, Tenth Revision (ICD-10). Since about $98 \%$ of the entire Korean population is covered by the national health insurance except for special circumstances, the claim data is a large-scale representative data reflecting the use of medical services by the entire South Korean population. With this database, it is possible to perform a longitudinal cohort study examining long-term outcomes. ${ }^{22)}$

In this study, we examined the duration of the first antipsychotic treatment in patients with schizophrenia, using the HIRA database. The re-initiation of treatment after treatment discontinuation was used as a proxy for relapse. Associations of the duration of the first antipsychotic treatment with the occurrence of treatment re-initiation and time to re-initiation of treatment were examined. This study has an advantage over previous studies in terms of using large-scale real-world data, thus minimizing selection bias.

\section{Methods}

\section{Study population}

Individuals were selected using the HIRA database from 2007 to 2016. For identifying patients with first-episode schizophrenia, the following criteria were applied: 1) diagnostic code of F $20^{5)}$ after January 1, 2008, and the diagnosis of F20 did not change throughout the first antipsychotic treatment period, 2) not having diagnostic code of F20 or antipsychotic prescription within 3 years prior to the diagnosis of schizophrenia, 3) antipsychotic should have been prescribed within 3 days from the diagnosis of schizophrenia, and 4) onset age between 18 and 65 years. Exclusion criteria included diagnoses that should not have been established before the diagnosis of schizophrenia, including moderate to severe intellectual disability, dementia, autism spectrum disorder, psychotic disorder due to another medical condition, substance-induced psychotic disorder, and substance intoxication with perceptual disturbances. This study was approved by the Institutional Review Board of Asan Medical Center (IRB 2019-0895). The requirement for informed consent was waived owing to the use of anonymous information.

\section{Exposure and outcome}

Treatment discontinuation was defined as no antipsychotic prescription within 28 days from the expected date of the next prescription. If a patient had been prescribed antipsychotics 28 days later the expected date of the next visit, we regarded it as treatment discontinuation. The first antipsychotic treatment episode was determined from the diagnosis of schizophrenia to the first treatment discontinuation or the end of the study period (Dec 31, 2016), whichever occurred first. Even though antipsychotic drugs might be changed during the treatment period, we considered it an antipsychotic treatment episode as long as more than 28 days of the treatment gap did not exist in that period. The re-initiation of antipsychotic treatment was used as a proxy for relapse and defined as the initiation of antipsychotic treatment following the treatment discontinuation. The dose of each prescribed antipsychotic was converted to an olanzapine equivalent dose using equations in a previous study. ${ }^{23)}$ The antipsychotics included in the present study were as follows: bromperidol, chlorpromazine, chlorprothixene, haloperidol, long-acting injectable (LAI) haloperidol, levomepromazine, molindone, nemonapride, perphenazine, pimozide, thiothixene, amisulpride, aripiprazole, LAI aripiprazole, blonanserin, clozapine, olanzapine, paliperidone, LAI paliperidone, quetiapine, risperidone, LAI risperidone, sul- 
piride, ziprasidone, and zotepine. The observation period started from the diagnosis of schizophrenia to the end of the study period (Dec 31, 2016).

\section{Statistical analysis}

Continuous and categorical variables are presented as means (standard deviations, SD) or numbers (\%). The first antipsychotic treatment episode was divided into four subgroups $(<1$ year, 1 to $<2$ years, 2 to $<5$ years, and $\geq 5$ years). Rate of the treatment discontinuation, duration from the diagnosis of schizophrenia to the treatment discontinuation, and duration from the treatment discontinuation to the re-initiation of treatment were calculated. To examine the relationship between

Table 1. Demographics and clinical characteristics of the study population

\begin{tabular}{lc}
\hline \multicolumn{1}{c}{ Variable } & $\begin{array}{c}\text { Total patients } \\
(\mathrm{n}=38,479)\end{array}$ \\
\hline Age of onset, mean (SD), years & $39.8(12.7)$ \\
Sex, male, $\mathrm{n}(\%)$ & $13,601(45.1)$ \\
Onset year, $\mathrm{n}(\%)$ & \\
2010 & $5,364(17.8)$ \\
2011 & $5,354(17.8)$ \\
2012 & $4,571(15.2)$ \\
2013 & $4,031(13.4)$ \\
2014 & $3,877(12.9)$ \\
2015 & $3,655(12.1)$ \\
2016 & $3,291(10.9)$ \\
Total observation period per person, & $2.9(2.2)$ \\
mean (SD), years & \\
Number of the treatment discontinuations & $1.9(2.1)$ \\
per person, mean (SD) & \\
\hline
\end{tabular}

SD, standard deviation the duration of the first antipsychotic treatment episode and the mean daily olanzapine equivalent dose in the treatment episode, linear regression analysis with covariates of age and sex was conducted. The Cox proportional hazard regression model and linear regression analysis were used to examine the association between the duration of the first antipsychotic treatment episode and the occurrence of and time to re-initiation of treatment. The following variables were included as covariates: age, sex, the mean daily antipsychotic dose in the treatment episode, and the onset year. We categorized the patients into four subgroups by their mean daily olanzapine equivalent dose in the treatment episode ( $<10 \mathrm{mg} /$ day, 10 to $<20 \mathrm{mg} /$ day, 20 to $<30 \mathrm{mg} /$ day, and $\geq 30 \mathrm{mg} /$ day). We repeated the analyses in each subgroup. A p-value of less than 0.05 was considered as statistically significant. All statistical analyses were performed using the R software, ver. 3.4.1 (R Development Core Team, Vienna, Austria).

\section{Results}

We selected a total of 30,143 patients with first-episode schizophrenia who met the selection criteria (Table 1). The mean of onset age was 39.84 (12.7) years, and 13,601 (45.1\%) patients were male, and 16,542 (54.9\%) patients were female. The mean of observation period was 2.9 (2.2) years, and the patients experienced an average of 1.9 (2.1) treatment discontinuations during the observation period.

Of the 30,143 patients with first-episode schizophrenia, $76.4 \%(\mathrm{n}=23,030)$ had treatment discontinuation. Among them, $74.2 \%(\mathrm{n}=17,086)$ of the patients re-initiated antipsychotic treatment after treatment discontinuation (Table 2 and Fig. 1).

Table 2. Time to the treatment discontinuation and re-initiation of treatment grouped by first treatment episode duration

\begin{tabular}{|c|c|c|c|c|c|}
\hline & \multicolumn{5}{|c|}{ Duration of the first treatment episode } \\
\hline & $<1$ year & 1 to $<2$ years & $2<$ to 5 years & $>5$ years & Total \\
\hline Number of patients, $\mathrm{n}(\%)$ & $20,639(68.5)$ & $3,592(11.9)$ & $4,085(13.6)$ & $1,827(6.1)$ & 30,143 \\
\hline $\begin{array}{l}\text { Number of patients with the treatment } \\
\text { discontinuation*, n (\%) }\end{array}$ & $18,703(90.6)$ & $2,430(67.7)$ & $1,738(42.5)$ & $159(8.7)$ & $23,030(76.4)$ \\
\hline \multicolumn{6}{|l|}{ Time to the treatment discontinuation ${ }^{\dagger}$} \\
\hline Observation period, person-days, mean (SD) & $96.7(93.2)$ & $520.5(104.1)$ & $1194.8(315.8)$ & $2152.5(206.4)$ & $420.6(599.5)$ \\
\hline Time to event, days, mean (SD) & $88.9(88.2)$ & $510.4(103.1)$ & $1099.1(284.9)$ & $2047.3(178.1)$ & $223.1(343.5)$ \\
\hline $\begin{array}{l}\text { Number of patients with treatment re-initiation }{ }^{\ddagger} \text {, } \\
n(\%)\end{array}$ & $13,813(73.9)$ & $1,881(77.4)$ & $1,296(74.6)$ & $96(60.4)$ & $17,086(74.2)$ \\
\hline \multicolumn{6}{|l|}{ Time to re-initiation of treatment\$ } \\
\hline Observation period, person-days, mean (SD) & $486.7(622.1)$ & $328.9(467.0)$ & $266.0(364.1)$ & $143.0(156.7)$ & $451.1(594.4)$ \\
\hline Time to re-initiation of treatment, days, mean (SD) & $255.3(347.4)$ & $167.2(233.4)$ & $126.5(178.8)$ & $81.1(83.2)$ & $234.9(328.4)$ \\
\hline
\end{tabular}


The proportions of the patients with treatment episodes of $<1$ year, 1 to $<2$ years, 2 to $<5$ years, and $\geq 5$ years were $68.5 \%$, $11.9 \%, 13.6 \%$, and $6.1 \%$, respectively. The rate of treatment discontinuation in the group with less than 1 year of first treatment episode was $90.6 \%$. The rate of treatment discontinuation tended to decrease with longer first treatment episode durations, and the rate of treatment discontinuation in the group with more than 5 years was $8.7 \%$. The rates of treatment reinitiation were $73.9 \%, 77.4 \%, 74.6 \%$, and $60.4 \%$ in the groups with first treatment episode durations of $<1$ year, 1 to $<2$ years, 2 to $<5$ years, and $\geq 5$ years, respectively. The mean of time to re-initiation of treatment decreased with an increase in the first antipsychotic treatment episode duration; the mean of time to re-initiation of treatment was 255.3 (347.4) days in patients with treatment durations of less than 1 year and 81.1 (83.2) days in the patients with treatment durations of more than 5 years.

The mean daily olanzapine equivalent dose of antipsychotics in the first antipsychotic treatment episode was positively associated with the length of the treatment episode $(\beta=0.822$, $\mathrm{p}<0.001$ ) (Table 3). With adjustment of the dose of antipsychotics in the treatment episode along with age, sex, onset year, as the duration of the first treatment episode was longer, the time to re-initiation of treatment was shown to decrease significantly $(\beta=-0.146, p<0.001)$. Cox proportional hazard regression model with re-initiation of treatment as a categori-
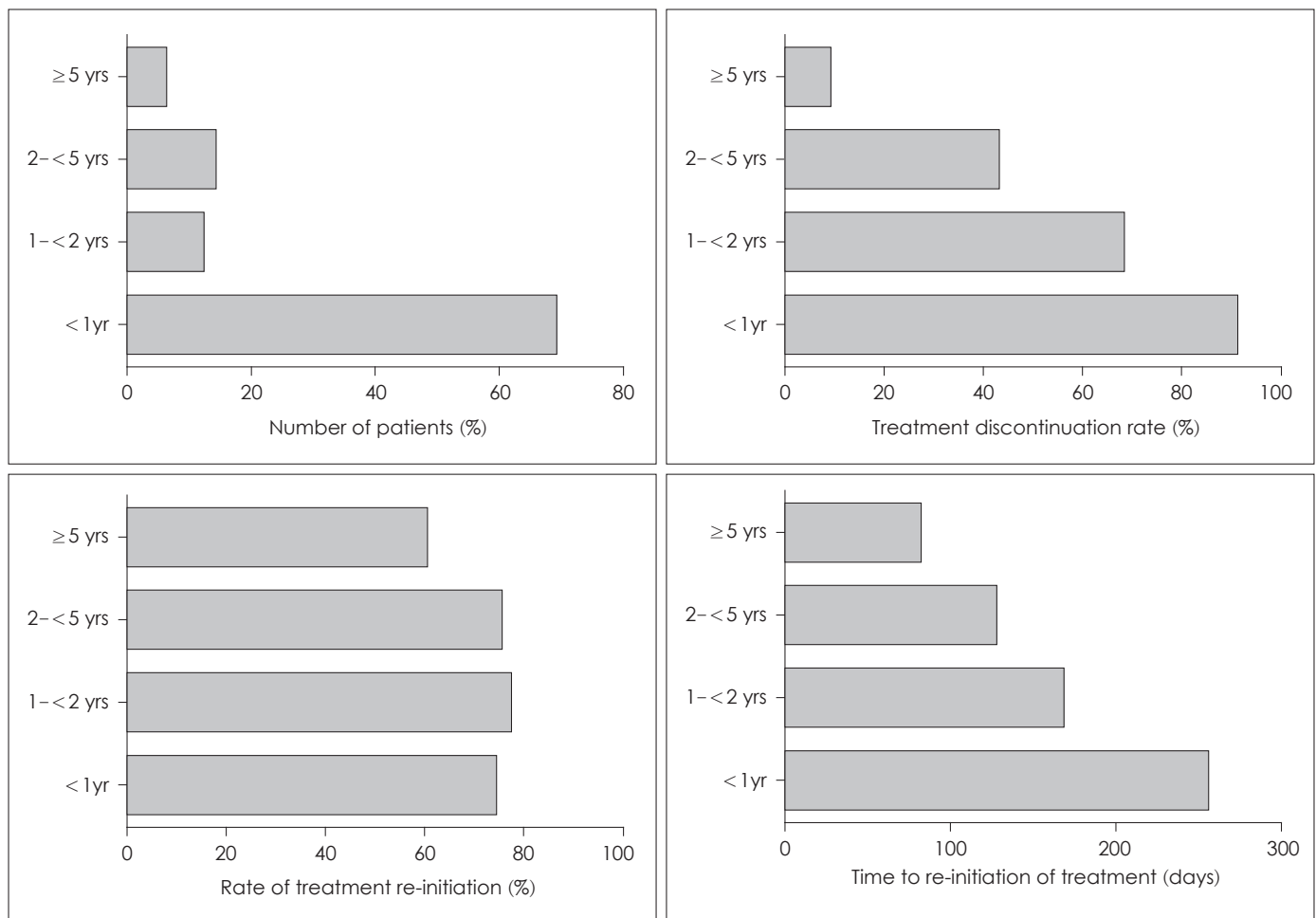

Fig. 1. Bar plots showing the number of patients, treatment discontinuation rate, rate of treatment re-initiation, and time to re-initiation of treatment by the duration of the first treatment episode. Y-axis indicates the duration of the first treatment episode.

Table 3. Relationships between the first treatment episode duration and time to re-initiation of treatment

\begin{tabular}{|c|c|c|c|c|c|c|c|c|}
\hline \multirow[t]{2}{*}{ Variable } & \multicolumn{3}{|c|}{$\begin{array}{l}\text { Duration of the first } \\
\text { treatment episode* }\end{array}$} & \multicolumn{3}{|c|}{$\begin{array}{l}\text { Time to re-initiation } \\
\text { of treatment }^{\dagger}\end{array}$} & \multicolumn{2}{|l|}{$\begin{array}{l}\text { Re-initiation } \\
\text { of treatment }^{\dagger}\end{array}$} \\
\hline & $\beta$ & $t$ & $p$ & $\beta$ & $t$ & $p$ & $\mathrm{HR}(95 \% \mathrm{Cl})$ & $p$ \\
\hline Age & 0.937 & 5.213 & $<0.001$ & 1.139 & 5.723 & $<0.001$ & $0.997(0.996-0.998)$ & 0.001 \\
\hline Sex & 0.778 & 0.171 & 0.865 & -15.246 & -3.047 & 0.002 & $1.037(1.006-1.069)$ & 0.020 \\
\hline $\begin{array}{l}\text { Mean daily olanzapine equivalent } \\
\text { dose in the first treatment episode }\end{array}$ & 0.822 & 6.732 & $<0.001$ & -1.194 & -8.839 & $<0.001$ & $1.006(1.005-1.006)$ & $<0.001$ \\
\hline Duration of the first treatment episode & - & - & - & -0.146 & -19.826 & $<0.001$ & $1.001(1.001-1.001)$ & $<0.001$ \\
\hline
\end{tabular}

*: Association with the mean daily olanzapine equivalent dose in the first treatment episode was evaluated with covariates of age, sex, and onset year; $\dagger$ : Association with the duration of the first treatment episode was evaluated with covariates of age, sex, mean daily olanzapine equivalent dose in the first treatment episode, and onset year 
cal variable showed that the association between the duration of the first treatment episode and re-initiation of treatment was significant, but close to 1.000 (hazard ratio $[\mathrm{HR}]=1.001$, $\mathrm{p}<0.001)$. We also repeated the above analyses within each subgroup, which was divided by the mean daily antipsychotic dose in the treatment episode (Appendix Tables 1 and 2). The negative relationship between the time to re-initiation of treatment and treatment episode duration and the HR of the duration of the treatment episode for re-initiation of treatment were significant in all subgroups except for those with a mean daily olanzapine equivalent dose of antipsychotics above $30 \mathrm{mg}$.

\section{Discussion}

We investigated the duration of the first antipsychotic treatment and re-initiation of treatment in first-episode schizophrenia, using a nationwide population claim database. Of the 30,143 patients with first-episode schizophrenia, a substantial proportion $(80.4 \%)$ had the first antipsychotic treatment episode of less than two years. The re-initiation of treatment occurred in $74.2 \%$ of the patients with the treatment discontinuation. As the duration of the treatment episode increased, the time to re-initiation of treatment showed a decreasing trend, but the rate of treatment re-initiation was relatively constant. A negative relationship was found between the duration of the treatment episode and the time to re-initiation of treatment. The duration of the treatment episode was also associated with the occurrence of treatment re-initiation, however which was not a notable finding.

In this study, most of the patients $(80.4 \%, n=24,231)$ had the first antipsychotic treatment duration of less than 2 years. According to a previous study, $40 \%-50 \%$ of patients with firstepisode schizophrenia discontinue antipsychotic treatment within 1-2 years from the onset of the disease. ${ }^{24)}$ A recent systematic review reported that most treatment guidelines for schizophrenia recommend continuing the antipsychotic treatment for 1-2 years after the stabilization of acute symptoms in first-episode schizophrenia. ${ }^{20)}$ However, previous studies ${ }^{25-27)}$ have reported that a substantial proportion of patients discontinue the antipsychotic treatment earlier than the recommended time point in clinical practice. The current study also reported a higher proportion of patients discontinuing treatment within 2 years compared to those reported in previous studies. Given that early discontinuation of the antipsychotic drugs could lead to poor clinical outcome in schizophrenia, ${ }^{15)}$ further strategies to improve the treatment compliance in the early phase of schizophrenia are required.

We observed that $74.2 \%$ of the patients who discontinued the antipsychotic treatment showed the re-initiation of treatment, which is consistent with previous findings where 57 to $98 \%$ of patients with first-episode schizophrenia experiences relapse after the treatment discontinuation. ${ }^{3-6)}$ The present study showed that the HR of the duration of the treatment episode for the occurrence of treatment re-initiation was statistically significant but close to 1.000 . The same results were observed in the subgroup analyses in which the patients were categorized into four subgroups based on their mean daily antipsychotic dose. A recent study using the Finland population database reported an increased risk of rehospitalization in patients with first-episode schizophrenia who discontinued antipsychotic drugs, compared to those who continued the medications. ${ }^{28)}$ The study also showed a higher risk of rehospitalization as the duration of the first antipsychotic treatment increased. Notably, the authors also found that the proportion of the patients having a treatment failure after the treatment discontinuation was relatively constant, regardless of the duration of the first antipsychotic treatment, which is consistent with the findings of our study.

In the present study, the patients who discontinued the antipsychotic treatment experienced re-initiation of treatment after an average of 234.9 days, which is similar to the results of a previous study. ${ }^{19)}$ As the duration of the treatment episode was increased, a decreasing trend was noted in the time to reinitiation of treatment; this corresponds to the results of a previous study. ${ }^{28)}$ One of the possible explanations is that the patients with longer treatment episode durations would be more severely ill, resulting in a shorter period between the treatment discontinuation and the re-initiation of treatment. We addressed this issue by including a covariate of the mean daily antipsychotic dose as a surrogate for the severity of the patients' symptoms and performing the subgroup analyses which categorized the patients by their mean daily antipsychotic dose. The subgroup analyses showed similar findings to those from the analyses with the entire cohort, except for those with a mean daily olanzapine equivalent dose of antipsychotics above 30 mg. The significant association of the treatment episode duration with the time to re-initiation of treatment tended to decrease as the mean daily antipsychotic dose increased. The relatively constant rate of treatment re-initiation across different treatment episode durations also declined the possibility of the effect of illness severity. If the longer first treatment episode duration and the shorter time to re-initiation of treatment was due to the severity of illness, the rate of treatment 
re-initiation would have increased with an increase in the first treatment episode duration. The current findings could be partially explained by the dopamine hypersensitivity hypothesis by Murray et al. ${ }^{29)}$ who reported that a long-term use of antipsychotics leads to an upregulation of D2/D3 receptors and makes the patients more vulnerable to relapse. However, the mean of time to re-initiation of treatment in the present study was longer than 6 weeks, which was not explainable by the dopamine hypersensitivity hypothesis.

This nationwide cohort study covered all patients of interest, and not just patients who complied with limited conditions, thus providing a representative finding. Because the patients were obligated to have national medical insurance, it was possible to follow up with the patients without loss. However, some limitations should be considered in the interpretation of the findings. First, it was uncertain whether the treatment discontinuation occurred due to clinician's decision or patients' non-compliance. In addition, owing to inherent limitations of the HIRA database, we could not investigate why some patients continued the antipsychotic treatment over a long-term period, i.e., over 5 years. Second, because we considered the re-initiation of antipsychotic treatment after treatment discontinuation as relapse, relapse such as aggravation of psychiatric symptoms or hospitalization during the continuation of treatment was not considered in the present study. In real-clinical practice, it is not uncommon for patients to experience relapse even with the continuation of antipsychotic treatment. ${ }^{28,30)}$ The reason for using the re-initiation of antipsychotic treatment as a marker for relapse was that when a patient relapses in no use of antipsychotic drugs, psychiatric hospitalization is usually indicated, however, in some cases, the re-initiation of antipsychotic treatment could lead to improvement of psychiatric symptoms to the degree to which psychiatric hospitalization is no longer needed. Third, the mean onset age of schizophrenia in the current study was higher than the ordinary onset age of schizophrenia. This study was conducted using the claim database and the medical records of individual patients could not be retrieved, which hampered the decision of whether the diagnosis of patients was first-onset schizophrenia or not. Fourth, even though we adopted the mean daily olanzapine equivalent dose as a measure for the severity of psychiatric symptoms, the mean daily antipsychotic dose might not exactly reflect the severity of psychiatric symptoms. It might be possible that clinicians were not able to increase dose of antipsychotics because of adverse effects or paranoid tendencies of patients.

\section{Conclusion}

In this study, we investigated the association between the duration of the first antipsychotic treatment and re-initiation of treatment in schizophrenia. Most patients had been prescribed antipsychotic drugs for less than 2 years after the disease onset. Long-term antipsychotic treatment was not associated with the rate of treatment re-initiation but had a negative association with the time to re-initiation of treatment. Further research is needed to verify the current findings and obtain the optimal duration of the maintenance treatment in firstepisode schizophrenia while maintaining a balance between the risk of relapse and adverse effects because of a long-term use of antipsychotics.

Keywords: Antipsychotic treatment; Re-initiation of treatment; Schizophrenia.

\section{ORCID iDs}

Minho Song

Jungsun Lee

Harin Kim

Soojin Ahn

Young Jae Choi

Young Tak Jo

Sung Woo Joo

\begin{abstract}
https://orcid.org/0000-0002-8819-1790
https://orcid.org/0000-0003-2171-2720

https://orcid.org/0000-0002-7304-4616

https://orcid.org/0000-0002-4413-5025

https://orcid.org/0000-0001-7299-6976

https://orcid.org/0000-0002-0561-2503

https://orcid.org/0000-0001-6555-9110
\end{abstract}

\section{REFERENCES}

1) McGrath J, Saha S, Chant D, Welham J. Schizophrenia: a concise overview of incidence, prevalence, and mortality. Epidemiol Rev 2008;30:67-76.

2) Robinson D, Woerner MG, Alvir JM, Bilder R, Goldman R, Geisler $\mathrm{S}$, et al. Predictors of relapse following response from a first episode of schizophrenia or schizoaffective disorder. Arch Gen Psychiatry 1999;56:241-247.

3) Gitlin M, Nuechterlein K, Subotnik KL, Ventura J, Mintz J, Fogelson DL, et al. Clinical outcome following neuroleptic discontinuation in patients with remitted recent-onset schizophrenia. Am J Psychiatry 2001;158:1835-1842.

4) Boonstra G, Burger H, Grobbee DE, Kahn RS. Antipsychotic prophylaxis is needed after remission from a first psychotic episode in schizophrenia patients: results from an aborted randomised trial. Int J Psychiatry Clin Pract 2011;15:128-134.

5) Gaebel W, Riesbeck M, Wolwer W, Klimke A, Eickhoff M, von Wilmsdorff $\mathrm{M}$, et al. Relapse prevention in first-episode schizophrenia: maintenance vs intermittent drug treatment with prodromebased early intervention: results of a randomized controlled trial within the German Research Network on Schizophrenia. J Clin Psychiatry 2011;72:205-218.

6) Emsley R, Oosthuizen PP, Koen L, Niehaus DJ, Martinez G. Symptom recurrence following intermittent treatment in first-episode schizophrenia successfully treated for 2 years: a 3-year open-label clinical study. J Clin Psychiatry 2012;73:e541-547.

7) McCreadie RG, Wiles D, Grant S, Crockett GT, Mahmood Z, Livingston MG, et al. The Scottish first episode schizophrenia study: VII. two-year follow-up. Acta Psychiatr Scand 1989;80:597-602. 
8) Kane JM. Treatment strategies to prevent relapse and encourage remission. J Clin Psychiatry 2007;68 Suppl 14:27-30.

9) Wyatt RJ. Research in schizophrenia and the discontinuation of antipsychotic medications. Schizophr Bull 1997;23:3-9.

10) Lieberman JA, Alvir JM, Koreen A, Geisler S, Chakos M, Sheitman $\mathrm{B}$, et al. Psychobiologic correlates of treatment response in schizophrenia. Neuropsychopharmacology 1996;14:13S-21S.

11) Alvarez-Jimenez M, Priede A, Hetrick SE, Bendall S, Killackey E, Parker AG, et al. Risk factors for relapse following treatment for first episode psychosis: a systematic review and meta-analysis of longitudinal studies. Schizophr Res 2012;139:116-128.

12) De Hert M, Sermon J, Geerts P, Vansteelandt K, Peuskens J, Detraux J. The use of continuous treatment versus placebo or intermittent treatment strategies in stabilized patients with schizophrenia: a systematic review and meta-analysis of randomized controlled trials with first- and second-generation antipsychotics. CNS Drugs 2015;29:637-658.

13) Leucht S, Tardy M, Komossa K, Heres S, Kissling W, Salanti G, et al. Antipsychotic drugs versus placebo for relapse prevention in schizophrenia: a systematic review and meta-analysis. Lancet 2012; 379:2063-2071.

14) Takeuchi H, Kantor N, Sanches M, Fervaha G, Agid O, Remington G. One-year symptom trajectories in patients with stable schizophrenia maintained on antipsychotics versus placebo: meta-analysis. Br J Psychiatry 2017;211:137-143.

15) Hui CLM, Honer WG, Lee EHM, Chang WC, Chan SKW, Chen ESM, et al. Long-term effects of discontinuation from antipsychotic maintenance following first-episode schizophrenia and related disorders: a 10 year follow-up of a randomised, double-blind trial. Lancet Psychiatry 2018;5:432-442.

16) Sampson S, Mansour M, Maayan N, Soares-Weiser K, Adams CE. Intermittent drug techniques for schizophrenia. Cochrane Database Syst Rev 2013:CD006196.

17) Newcomer JW. Metabolic considerations in the use of antipsychotic medications: a review of recent evidence. J Clin Psychiatry 2007; 68 Suppl 1:20-27.

18) Bostwick JR, Guthrie SK, Ellingrod VL. Antipsychotic-induced hyperprolactinemia. Pharmacotherapy 2009;29:64-73.

19) Emsley R, Chiliza B, Asmal L, Harvey BH. The nature of relapse in schizophrenia. BMC Psychiatry 2013;13:50

20) Shimomura Y, Kikuchi Y, Suzuki T, Uchida H, Mimura M, Takeuchi H. Antipsychotic treatment in the maintenance phase of schizophrenia: an updated systematic review of the guidelines and algorithms. Schizophr Res 2020;215:8-16.

21) Dans AL, Dans LF, Guyatt GH, Richardson S. Users' guides to the medical literature: XIV. how to decide on the applicability of clinical trial results to your patient. JAMA 1998;279:545-549.

22) Kim JA, Yoon S, Kim LY, Kim DS. Towards actualizing the value potential of Korea health insurance review and assessment (HIRA) data as a resource for health research: strengths, limitations, applications, and strategies for optimal use of HIRA data. J Korean Med Sci 2017;32:718-728.

23) Gardner DM, Murphy AL, O’Donnell H, Centorrino F, Baldessarini RJ. International consensus study of antipsychotic dosing. Am J Psychiatry 2010;167:686-693.

24) Sadock BJ, Sadock VA, Ruiz P. Kaplan \& Sadock's synopsis of psychiatry: behavioral sciences/clinical psychiatry. 11th ed. Philadelphia: Wolters Kluwer;2015.

25) Barkhof E, Meijer CJ, de Sonneville LM, Linszen DH, de Haan L. Interventions to improve adherence to antipsychotic medication in patients with schizophrenia: a review of the past decade. Eur Psychiatry 2012;27:9-18.

26) Haddad PM, Brain C, Scott J. Nonadherence with antipsychotic medication in schizophrenia: challenges and management strategies. Patient Relat Outcome Meas 2014;5:43-62.

27) Lieberman JA, Stroup TS, McEvoy JP, Swartz MS, Rosenheck RA, Perkins DO, et al. Effectiveness of antipsychotic drugs in patients with chronic schizophrenia. N Engl J Med 2005;353:1209-1223.

28) Tiihonen J, Tanskanen A, Taipale H. 20-year nationwide follow-up study on discontinuation of antipsychotic treatment in first-episode schizophrenia. Am J Psychiatry 2018;175:765-773.

29) Murray RM, Quattrone D, Natesan S, van Os J, Nordentoft M, Howes $\mathrm{O}$, et al. Should psychiatrists be more cautious about the longterm prophylactic use of antipsychotics? Br J Psychiatry 2016;209: 361-365.

30) Fenton WS, Blyler CR, Heinssen RK. Determinants of medication compliance in schizophrenia: empirical and clinical findings. Schizophr Bull 1997;23:637-651. 


\section{Appendix}

Table 1. Relationships between the first treatment episode duration and time to re-initiation of treatment by mean daily olanzapine equivalent dose of antipsychotics in the first treatment episode

\begin{tabular}{lccc}
\hline \multirow{2}{*}{ Group* } & \multicolumn{3}{c}{ Time to re-initiation of treatment ${ }^{\dagger}$} \\
\cline { 2 - 4 } & $\beta$ & $\dagger$ & $\mathrm{p}$ \\
\hline Group 1 $(<10 \mathrm{mg} /$ day $)$ & -0.156 & -15.278 & $<0.001$ \\
Group 2 $(10 \mathrm{mg} /$ day & -0.141 & -10.731 & $<0.001$ \\
to $<20 \mathrm{mg} / \mathrm{day})$ & & & \\
Group $3(20 \mathrm{mg} /$ day & -0.093 & -3.646 & $<0.001$ \\
to $<30 \mathrm{mg} / \mathrm{day})$ & & & \\
Group 4 ( $230 \mathrm{mg} /$ day $)$ & -0.025 & -0.936 & 0.350 \\
\hline
\end{tabular}

$*$ : Categorized by mean daily olanzapine equivalent dose of antipsychotics in the first treatment episode; $\dagger$ : Association with the duration of the first treatment episode was evaluated with covariates of age, sex, mean daily olanzapine equivalent dose in the first treatment episode, and onset year
Table 2. Relationships between the first treatment episode duration and re-initiation of treatment by mean daily olanzapine equivalent dose of antipsychotics in the first treatment episode

\begin{tabular}{lcc}
\hline \multirow{2}{*}{ Group* } & \multicolumn{2}{c}{ Re-initiation of treatment ${ }^{\dagger}$} \\
\cline { 2 - 3 } & $\mathrm{HR}(95 \% \mathrm{Cl})$ & $\mathrm{p}$ \\
\hline Group 1 (<10 mg/day) & $1.001(1.001-1.001)$ & $<0.001$ \\
Group 2 $(10 \mathrm{mg} /$ day & $1.001(1.001-1.001)$ & $<0.001$ \\
to $<20 \mathrm{mg} / \mathrm{day})$ & & \\
Group 3 $(20 \mathrm{mg} /$ day & $1.000(1.000-1.001)$ & $<0.001$ \\
to $<30 \mathrm{mg} /$ day $)$ & $1.000(1.000-1.000)$ & 0.305 \\
Group 4 $(\geq 30 \mathrm{mg} /$ day $)$ &
\end{tabular}

*: Categorized by mean daily olanzapine equivalent dose of antipsychotics in the first treatment episode; $\dagger$ : Association with the duration of the first treatment episode was evaluated with covariates of age, sex, mean daily olanzapine equivalent dose in the first treatment episode, and onset year 